\title{
Rifampicin Induced Acute Interstitial Nephritis in a Patient with Tubercular Lymphadenitis: A Case Report
}

\author{
Khan $\mathrm{HH}^{\mathrm{a}}$, Rahim MA ${ }^{\mathrm{b}}$, Ananna MA ${ }^{\mathrm{b}}$,Chowdhury $\mathrm{TA}^{\mathrm{c}}$, Iqbal $\mathrm{S}^{\mathrm{d}}$
}

\begin{abstract}
Rifampicin is one of the most effective anti-tubercular agents. Among its rare adverse effects, acute interstitial nephritis (AIN) is noteworthy. Here, we describe the case history of a 55-year-old female with tubercular lymphadenitis who developed rifampicin induced AIN upon re-exposure and recovered satisfactorily without requiring steroids. Rifampicin induced AIN should be kept in mind when patients present with acute kidney injury as prompt diagnosis and discontinuation of the drug has excellent prognosis.
\end{abstract}

Key words: acute interstitial nephritis, rifampicin, tuberculosis.

(BIRDEM Med J 2018; 8(3): 257-259)

\section{Introduction}

Tuberculosis (TB) is a highly prevalent disease in developing countries like Bangladesh. As such the rare side effects of anti-tubercular (anti-TB) drugs are not so uncommon. Rifampicin is one of the key drugs that helped to shorten the treatment duration in TB. Acute interstitial nephritis (AIN) as a complication of antiTB therapy has been most commonly reported due to rifampicin. ${ }^{1}$ According to the American Thoracic Society Guidelines, physicians are not currently required to monitor renal function during the course of anti-TB treatment unless the patient is at high risk for hepatic or renal abnormalities. Although it typically occurs in patients receiving intermittent rifampicin therapy, cases occurring during continuous treatment have also been reported. ${ }^{2}$ Here, we describe the case history of a patient who developed rifampicin induced AIN upon reexposure to the drug.

\section{Author Information}

a. Dr. Hafsa Hassan Khan, MD Resident, BIRDEM, Dhaka, Bangladesh

b. Dr. Muhammad Abdur Rahim, Mehruba Alam Ananna, Assistant Professor, Department of Nephrology, BIRDEM, Dhaka, Bangladesh

c. Dr. Tufayel Ahmed Chowdhury, Registrar, Department of Nephrology, BIRDEM, Dhaka, Bangladesh

d. Dr. Sarwar Iqbal, Professor, Department of Nephrology, BIRDEM, Dhaka, Bangladesh

Address of correspondence: Dr. Hafsa Hassan Khan, MD Resident, BIRDEM, Dhaka, Bangladesh, E-mail: hafsa.hassan135@gmail.com Received: August 10, 2018

Accepted: August 12, 2018

\section{Case Report}

A 55-year-old lady, known case of diabetes mellitus, hypertension and chronic kidney disease (CKD) stage 5 (serum creatinine $4.1 \mathrm{mg} / \mathrm{dl}$ ) with a past history of smear positive pulmonary TB, treated for 6 months with anti-TB drugs, presented with a 2-month history of low grade fever and multiple nodular swelling in right axilla. Fine needle aspiration cytology (FNAC) was suggestive of tubercular lymphadenitis (Figure 1) and she was started on modified category II anti-TB therapy

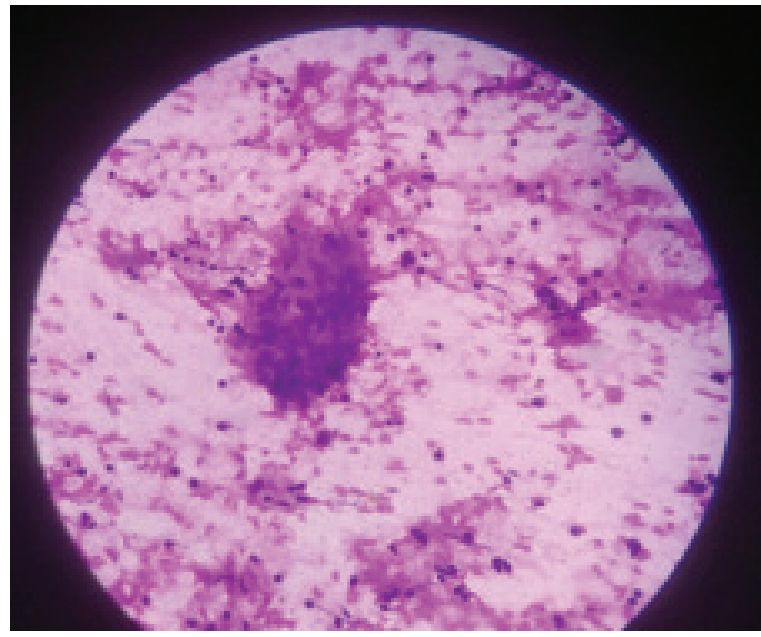

Figure 1 Fine needle aspiration cytology from axillary lymph node showing scattered as well as aggregates of epitheloid cells and clumps of caseous necrotic material, a good number of lymphocytes, histiocytes and some multinucleated giant cells; suggestive of tubercular lymphadenitis 
comprising isoniazid, rifampicin, pyrazinamide, ethambutol and moxifloxacin as daily therapy.

However, six weeks later she was admitted with the complaints of loss of appetite, occasional vomiting and decreased urine output. There was no jaundice or any obvious cause of her symptoms. Anti-TB therapy induced acute kidney injury(AKI) was suspected and all drugs were withheld.

Blood tests revealed hemoglobin $8.1 / \mathrm{g} / \mathrm{dl}$, white cells $8450 / \mathrm{mm}^{3}$ with increased eosinophil (18.7\%). Serum creatinine was found to be markedly elevated from baseline value of $4.1 / \mathrm{mg} / \mathrm{dl}$, prior to starting anti-TB therapy, to $13.7 \mathrm{mg} / \mathrm{dl}$. Urinalysis revealed sterile leucocyturia and eosinophiluria $(2 \%$, normal $<1 \%)$. Blood film showed features of hemolysis and eosinophilia. Reticulocyte count was increased (8\%), but Coomb's test was negative. Her liver biochemistry remained normal.

We diagnosed her as acute kidney injury (AKI) on CKD due to rifampicin induced AIN. We did not do renal biopsy in our patient due to small kidneys (right kidney $82 \mathrm{~mm}$, left kidney $84 \mathrm{~mm}$ ). Patient was advised hemodialysis but she refused. As the patient was clinically and hemodynamically stable with no features of acute pulmonary edema, we continued to monitor her renal function. Serum creatinine gradually began to decline and urine output gradually began to increase after stopping anti-TB medications (Figure 2).

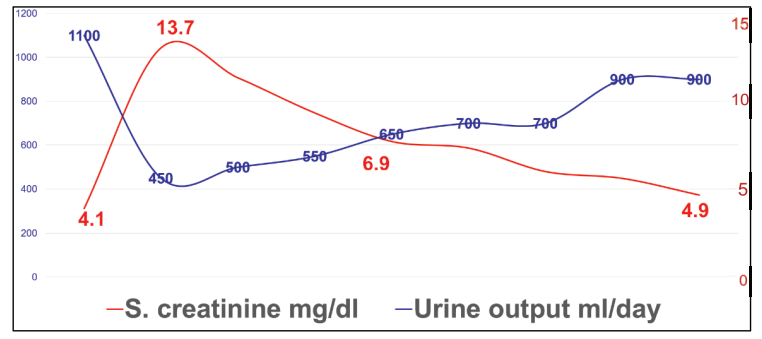

Figure 2 Increasing urine output with improving renal function

After two weeks, anti-TB drugs were reintroduced excluding rifampicin and there was no further deterioration of renal function. Serum creatinine returned to near baseline value without use of steroid (Table 1). Eosinophilia also resolved with time.

\section{Discussion}

AIN is an important cause of AKI resulting from immune-mediated tubulointerstitial injury, commonest cause being drugs. Despite being uncommon, rifampicin is the most frequent anti-TB medication associated with AIN. It is suggested that rifampicin functions as a molecule which, when bound to proteins, elicits an immune response by creating anti-rifampicin antibodies. It usually occurs in patients who have either previously taken the drug or received intermittent treatment. AKI occurs immediately after rifampicin re-administration, even if the episode is several months or years after the first treatment. ${ }^{3}$ Our patient had a history of taking rifampicin about eight years ago. One hypothesized mechanism for this is that, a quantity of antibody accumulates during a break in rifampicin treatment when there is absence of the offending antigen. When rifampicin is restarted, an immune reaction of a higher order of magnitude occurs due to the enhanced stores of circulating antibodies. ${ }^{4}$

The mechanism of rifampin-induced AKI is not well established. Several studies suggest that it is either a type II or type III hypersensitivity reaction induced by rifampin antigens which form immune complexes that are deposited in renal vessels, the glomerular endothelium and the interstitial area. ${ }^{5}$ Rifampicin dependent antibodies against the I-antigen which is present on erythrocytes and also on tubular epithelial cells is a possible explanation of the commonly associated hemolytic anaemia. ${ }^{6}$

It is important to make a prompt diagnosis of AIN in order to prevent further kidney damage. Although nonspecific, complete blood count to assess for eosinophilia and anemia and urinalysis to detect eosinophiluria can be done, both of which were present in our case. The specific time-course of events, in association with absence of other potential causes for AKI, established rifampicin as the sole aetiology in the current case. Thus, testing for anti-rifampicin antibody, which is not

Table I Patient's renal function during course of treatment

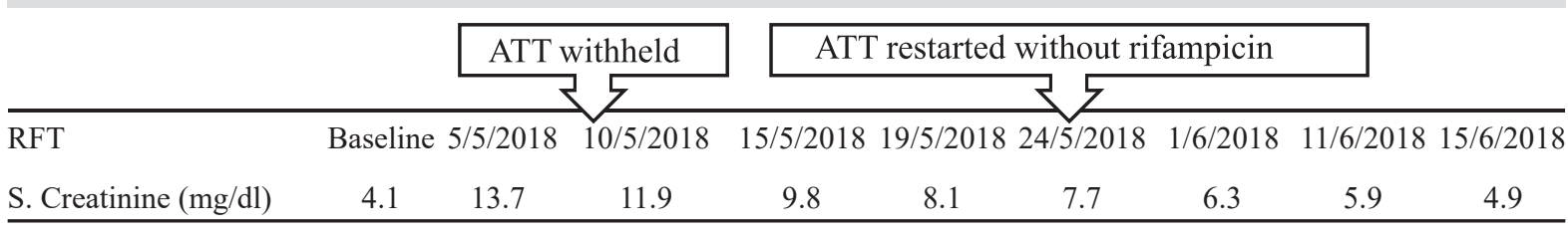


available in our country, was not considered necessary in our patient. Also post rifampicin AKI cases without demonstrable circulating antibodies have been described.

A definitive diagnosis of AIN should be made by renal biopsy but it may not be necessary in those who have clearly documented onset of renal failure after initiation of a culprit drug and improve immediately upon stopping the offending agent. ${ }^{7}$ In our patient renal biopsy could not be done due to small kidneys and as her renal function returned to near baseline value upon discontinuation of rifampicin, it was not considered necessary. Biopsy, if done, typically shows plasma and lymphocytic infiltrates in peri-tubular areas of the interstitium, but features of acute tubular necrosis are also common.

Once a diagnosis of AIN is established, the offending medication should be discontinued as soon as possible. Although controversial, there is some evidence suggesting that corticosteroid therapy accelerates renal recovery in patients diagnosed with drug-induced $\mathrm{AIN}^{8}$ whereas other studies revealed no significant difference. ${ }^{9}$ Our patient improved satisfactorily without use of steroids. Cruz DN and Perazella MA described an algorithm for the diagnosis, management and use of steroid in AIN. 10

The treatment of TB can be complicated in the setting of AIN. If rifampicin is not tolerated, the duration of treatment of TB is a minimum of 12 months, even for drug susceptible pulmonary TB. ${ }^{11}$ In our patient, an alternative regimen for the treatment of tubercular lymphadenitis consisting of isoniazid, pyrazinamide, ethambutol and moxifloxacin was started and the initial response was satisfactory.

The outcome of rifampicin-induced AIN is favorable in a majority of cases. Patients who discontinue the offending medications within two weeks of the onset of AIN are more likely to recover nearly baseline renal function than those who remain on the precipitating medication for three or more weeks. ${ }^{12}$

\section{Conclusion}

AIN should be borne in mind when patients on rifampicin develop deterioration of renal function. Early diagnosis and discontinuation of rifampicin are of fundamental importance for recovering renal function.

Conflict of interest: Nothing to declare.

\section{References}

1. Rudraraju M. Rifampin Induced Acute Tubulo-interstitial Nephritis: Rare Event of Commonly Used Drug. Journal of Case Reports 2017;7:133-35.

2. Afroz F, Hossain MD, Ahmed JU, Haque WMM. Rifampicin Induced Acute Interstitial Nephritis And Exfoliative Dermatitis Complicating Pulmonary Tuberculosis - A Case Report.BIRDEM Med J 2017;7:168-71.

3. Covic A, Goldsmith DJA, Segall L, Stoicescu C, Lungu S, Volovat $\mathrm{C}$, et al. Rifampicin-induced acute renal failure: a series of 60 patients. Nephrol Dial Transplant 1998;13:92429.

4. Di Berardino L, Perna G, Silvestri LG. Antibodies against rifampicin in patients with tuberculosis after discontinuation of daily treatment. Am Rev Respir Dis 1976;114:1189-90.

5. Muthukumar T, Jayakumar M, Fernando EM, Muthusethupathi MA. Acute renal failure due to rifampicin: a study of 25 patients. Am J kidney Dis 2002;14:690-96.

6. Acute interstitial nephritis during rifampicin therapy can be a paradoxical response: a case report Jan van der Meulen. Published in cases journal

7. Beck LH, Salant DJ. Tubulointerstitial diseases of the kidney. Harrison's Principles of Internal Medicine. $18^{\text {th }}$ ed. McGraw Hill Professional Publishing; 2012.p.2367-674.

8. González E, Gutiérrez E, Galeano C, Chevia C, deSequera P, Bernis $\mathrm{C}$, et al. Early steroid treatment improves the recovery of renal function in patients with drug-induced acute interstitial nephritis. Kidney Int 2008;73:940-46.

9. Clarkson MR, Giblin L, O'Connell FP, O'Kelly P, Walshe JJ, Conlon P, et al. Acute interstitial nephritis: clinical features and response to corticosteroid therapy. Nephrol Dial Transplant 2004;19:2778-83.

10. Cruz DN, Perazella MA. Drug-inducted acute tubulointerstitial nephritis: the clinical spectrum. Hosp Pract 1998;33:163

11. American Thoracic Society, CDC, and Infectious Diseases Society of America. Infectious diseases society of treatment of tuberculosis. MMWR Recomm Rep 2003;52(RR-11):1e77.

12. Kodner CM, Kudrimoti A. Diagnosis and Management of Acute Interstitial Nephritis. Am Fam Physician 2003;67:252734. 